\title{
Elixhauser comorbidity measures-based risk factors associated with 30-day mortality in elderly population after femur fracture surgery: a propensity score- matched retrospective case-control study
}

\author{
Dohyung Kim, Hyunmin Jo, Younsuk Lee, Kyoung Ok Kim \\ Department of Anesthesiology and Pain Medicine, Dongguk University Ilsan Hospital, Goyang, Korea
}

Background: As the average life expectancy increases, anesthesiologists confront unique challenges in the perioperative care of elderly patients who have significant comorbidities. In this study, we evaluated Elixhauser comorbidity measures-based risk factors associated with 30day mortality in patients aged 66 years and older who underwent femur fracture surgery. Methods: We used the Medical Information Mart for Intensive Care III which contains the medical records of patients admitted to the intensive care unit (ICU) at Beth Israel Deaconess Medical Center in the United States between 2001 and 2012 to identify patients admitted to the ICU after femur fracture surgery $(n=209)$. Patients who died within 30 days of admission (case group, $n=49$ ) were propensity score-matched to patients who did not (control group, $\mathrm{n}=98$ ). The variables for matching were age, sex, race, anemia (hemoglobin $\leq 10 \mathrm{~g} / \mathrm{dl}$ ), and malignancy. We attempted to explain mortality via nine independent factors: hypertension, uncomplicated diabetes, complicated diabetes, congestive heart failure (CHF), cardiac arrhythmias, chronic pulmonary disease, renal failure, neurological disorders other than paralysis, and peripheral vascular disease.

Results: Logistic regression identified three significant risk factors: CHF, arrhythmias, and neurological disorders other than paralysis. The odds ratio (OR) for the 30-day mortality of CHF was 4.99 (95\% confidence interval [CI], 2.18 to 12.06). The equivalent ORs for cardiac arrhythmias and neurological disorders other than paralysis were $2.61(95 \% \mathrm{Cl}, 1.14$ to 6.21$)$ and 2.40 (95\% $\mathrm{Cl}, 0.95$ to 6.48$)$, respectively.

Conclusions: Identifying patients with these risk factors ( $\mathrm{CHF}$, arrhythmias, and neurological disorders other than paralysis) will assist clinicians with perioperative planning and provide caregivers with valuable information for decision-making.

Key Words: elderly; femur fracture; mortality; orthopedic; risk factor

\section{INTRODUCTION}

As the average life expectancy has increased, the population of elderly patients has also increased. This tendency is particularly noticeable in patients with femur fractures because femur fractures are associated with aging and osteoporosis, and can occur from minor injuries.

\section{Original Article}

Received: November 6, 2019

Revised: December 23, 2019

Accepted: February 4, 2020

Corresponding author

Kyoung Ok Kim

Department of Anesthesiology and

Pain Medicine, Dongguk University

Ilsan Hospital, 27 Dongguk-ro,

Ilsandong-gu, Goyang 10326, Korea

Tel: +82-31-961-7874

Fax: +82-31-961-7864

E-mail: pissces00@gmail.com

Copyright () 2020 The Korean Society of Critical Care Medicine

This is an Open Access article distributed under the terms of Creative Attributions Non-Commercial License (https:// creativecommons.org/li-censes/by-nc/4.0/) which permits unrestricted noncommercial use, distribution, and reproduction in any medium, provided the original work is properly cited. 
The mortality rate is relatively high in elderly patients after femur fracture surgery $[1,2]$. These patients will likely be admitted to the intensive care unit (ICU) due to their comorbidities, and intensivists confront unique challenges involving their perioperative care. The prediction of risk factors for mortality is critical for intensivists, in order to conduct perioperative care planning with surgeons and to inform caregivers about various treatment options.

Many tools are available to predict postoperative mortality and prognosis for these patients according to their comorbidities [3-5]. There have been many reports regarding risk factors associated with outcomes after femur fracture surgery in heterogenous patient populations [6,7]. However, this study focused on risk assessment for a homogenous patient population admitted to the ICU after femur surgery. We evaluated the risk factors associated with 30-day mortality in elderly patients admitted to the ICU after femur fracture surgery based on the Elixhauser comorbidity measures.

\section{MATERIALS AND METHODS}

\section{Database}

We conducted a retrospective propensity score-matched, casecontrol study using the Medical Information Mart for Intensive Care III (MIMIC-III), which contains comprehensive, timestamped information. MIMIC-III data are Health Insurance Portability and Accountability Act of 1996 (HIPAA) compliant, and all investigators with data access (YL, KK) were approved by PhysioNet.

MIMIC-III contains deidentified health-related data from electronic medical ICU records associated with over 40,000 patients admitted to the ICU at Beth Israel Deaconess Medical Center in the United States between 2001 and 2012 [8]. Researchers must complete a training course in order to access the MIMIC-III database. The latest version of PostgreSQL and analytical software (such as R) are required to build MIMICIII and run statistical analysis in a local Postgres SQl database. The size of the MIMIC-III is so large that a free disk space size of 75 GB is mandatory, and $100 \mathrm{~GB}$ is recommended for optimal performance.

\section{Approach}

All records were screened to identify patients aged 66 years and older admitted to the ICU for postoperative care after femur surgery. Patients who died within 30 days of ICU admission (case group) were propensity score-matched to patients who survived to postoperative day 30 (control group). We chose

\section{KEY MESSAGES}

- The mortality rate was relatively high in patients aged 66 years and older after femur fracture surgery.

- Congestive heart failure was the most important risk factor for 30-day mortality.

- Identifying patients with high mortality risks will assist clinicians with perioperative planning and provide valuable information to patients and caregivers who make decisions concerning treatment strategy.

age, sex, race, malignancy, and anemia (hemoglobin $\leq 10 \mathrm{~g} / \mathrm{dl}$ ) on hospital admission as variables for matching the control and case groups. All these variables, except race, are indexed in the Nottingham Femur Fracture Score (NHFS), where age between 66 and 86 years is assigned 3 points, and an age older than 86 years is assigned 4 points, out of a total of 10 points [9]. We referred to the Elixhauser comorbidity measures to select relevant comorbidities that might influence mortality [10]. The Elixhauser comorbidity measures for each patient were generated from the original dataset.

\section{Statistics}

R software ver. 3.3.2 (R Foundation, Vienna, Austria) was used for the statistical analyses. The study outcome was 30-day mor-

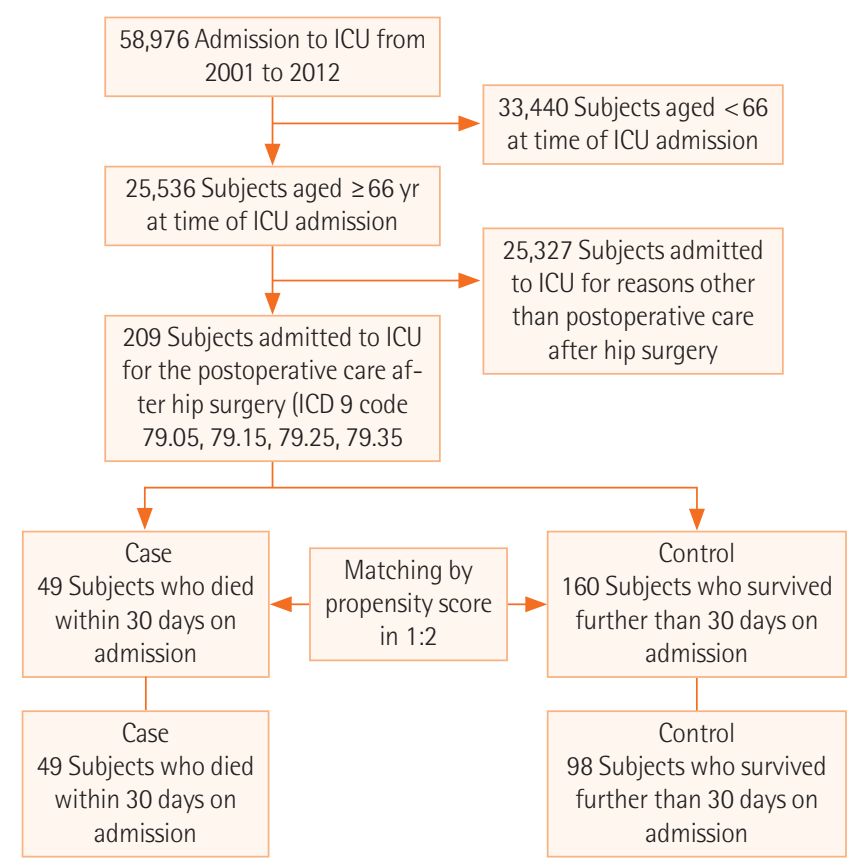

Figure 1. Flowchart of the study protocol. Case-control selection. ICU: intensive care unit; ICD 9: International Classification of Diseases, Ninth Revision. 
tality because deaths beyond that period might have less to do with the surgery itself and more to do with other illnesses and care services the patients received after they left the hospital. We aimed to explain mortality via nine independent factors selected from the Elixhauser comorbidity measures [10]. The measures we chose were hypertension, diabetes (uncomplicated), diabetes (complicated), congestive heart failure, cardiac arrhythmias, chronic pulmonary disease, renal failure, neurological disorders other than paralysis, and peripheral vascular disease. The significant factors were finally selected (using the criteria of $\mathrm{P}<0.10$ and low Akaike information criterion values) by logistic regression and a subsequent stepwise variable selection method. The odds ratios (ORs) were estimated for the significant factors under the $95 \%$ confidence intervals (CIs).

\section{RESULTS}

Figure 1 shows the flowchart of this study. There were records of 58,976 cases in the MIMIC-III data set. Of these cases, those under 66 years old were excluded $(n=33,440)$. After exclusion,

Table 1. ICD-9-CM procedure codes for femur operations

\begin{tabular}{ll}
\hline Code & \multicolumn{1}{c}{ Principal procedure } \\
\hline 79.05 & Closed reduction of fracture without internal fixation, femur \\
79.15 & Closed reduction of fracture with internal fixation, femur \\
79.25 & Open reduction of fracture without internal fixation, femur \\
79.35 & Open reduction of fracture with internal fixation, femur \\
\hline
\end{tabular}

ICD-9-CM: International Classification of Diseases, Ninth Revision, Clinical Modification. patients who had been admitted to the ICU for postoperative care after femur surgery (International Classification of Diseases, Ninth Revision, Clinical Modification [ICD-9-CM]; procedure code: 79.05, 79.15, 79.25, and 79.35) (Table 1) were enrolled in the study $(\mathrm{n}=209)$. Patients who died within 30 days of admission (case group, $\mathrm{n}=49$ ) were propensity score-matched to patients who did not (control group, $\mathrm{n}=98$ ). The patient characteristics and the number of comorbidities in the two groups are listed in Tables 2 and 3.

Of 209 patients who underwent femur fracture surgery, 49 patients died within 30 days of ICU admission (23.4\%). The overall 30-day mortality in the MIMIC-III data was $18.4 \%(4,696$ out of 25,536 ) in patients aged $\geq 66$ years. Three significant factors, congestive heart failure, arrhythmias, and neurological disorders other than paralysis were identified by logistic regression. The OR for the 30-day mortality of congestive heart failure was 4.99 (95\% CI, 2.18 to 12.06). The equivalent ORs for cardiac arrhythmias and neurological disorders other than paralysis were 2.61 (95\% CI, 1.14 to 6.21 ) and 2.40 (95\% CI, 0.95, 6.48), respectively (Figure 2).

Table 3. Numbers of patients according to comorbidity counts

\begin{tabular}{lcc}
\hline Number of comorbidities & $\begin{array}{c}\text { Case group } \\
(n=49)\end{array}$ & $\begin{array}{c}\text { Control group } \\
(n=160)\end{array}$ \\
\hline 0 & $1(2)$ & $12(8)$ \\
1 & $2(4)$ & $38(24)$ \\
2 & $13(27)$ & $42(26)$ \\
$\geq 3$ & $33(67)$ & $68(43)$ \\
\hline
\end{tabular}

Values are presented as number (\%) of patients.

Table 2. Demographic characteristics before and after the propensity score matching

\begin{tabular}{|c|c|c|c|c|c|c|}
\hline \multirow{2}{*}{ Variable } & \multicolumn{3}{|c|}{ Before propensity score matching } & \multicolumn{3}{|c|}{ After propensity score matching } \\
\hline & Case $(n=49)$ & Control $(n=160)$ & STD $^{\mathrm{a}}$ & Case $(n=49)$ & Control $(n=98)$ & STD \\
\hline \multicolumn{7}{|l|}{ Age (yr) } \\
\hline $66-85$ & $25(51.0)$ & $105(65.6)$ & -0.15 & $25(51.0)$ & $49(50.0)$ & 0.01 \\
\hline$\geq 86$ & $24(49.0)$ & $55(34.4)$ & 0.15 & $24(49.0)$ & $49(50.0)$ & -0.01 \\
\hline Male sex & $15(30.7)$ & $58(36.3)$ & -0.06 & $15(30.7)$ & $26(26.5)$ & 0.04 \\
\hline Race (white) & 45 (91.8) & $130(81.2)$ & 0.11 & 45 (91.8) & 88 (89.8) & 0.02 \\
\hline Metastatic cancer (yes) & 0 & $5(3.1)$ & -0.03 & 0 & 0 & 0.00 \\
\hline Solid tumor with-out metastasis & $1(2.0)$ & $3(1.9)$ & 0.00 & $1(2.0)$ & $1(1.0)$ & 0.01 \\
\hline Anemiab (yes) & $12(24.5)$ & $38(23.8)$ & 0.01 & $12(24.5)$ & $20(20.4)$ & 0.04 \\
\hline
\end{tabular}

Values are presented as number (\%) of patients.

STD: standardized difference.

${ }^{a}$ Case minus control: absolute differences in proportions divided by pooled standard deviation; $\mid$ STD $\mid>0.10$ is selected a priori for indicating imbalance;

${ }^{b}$ Plasma hemoglobin concentration $\leq 10 \mathrm{~g} / \mathrm{dl}$. 


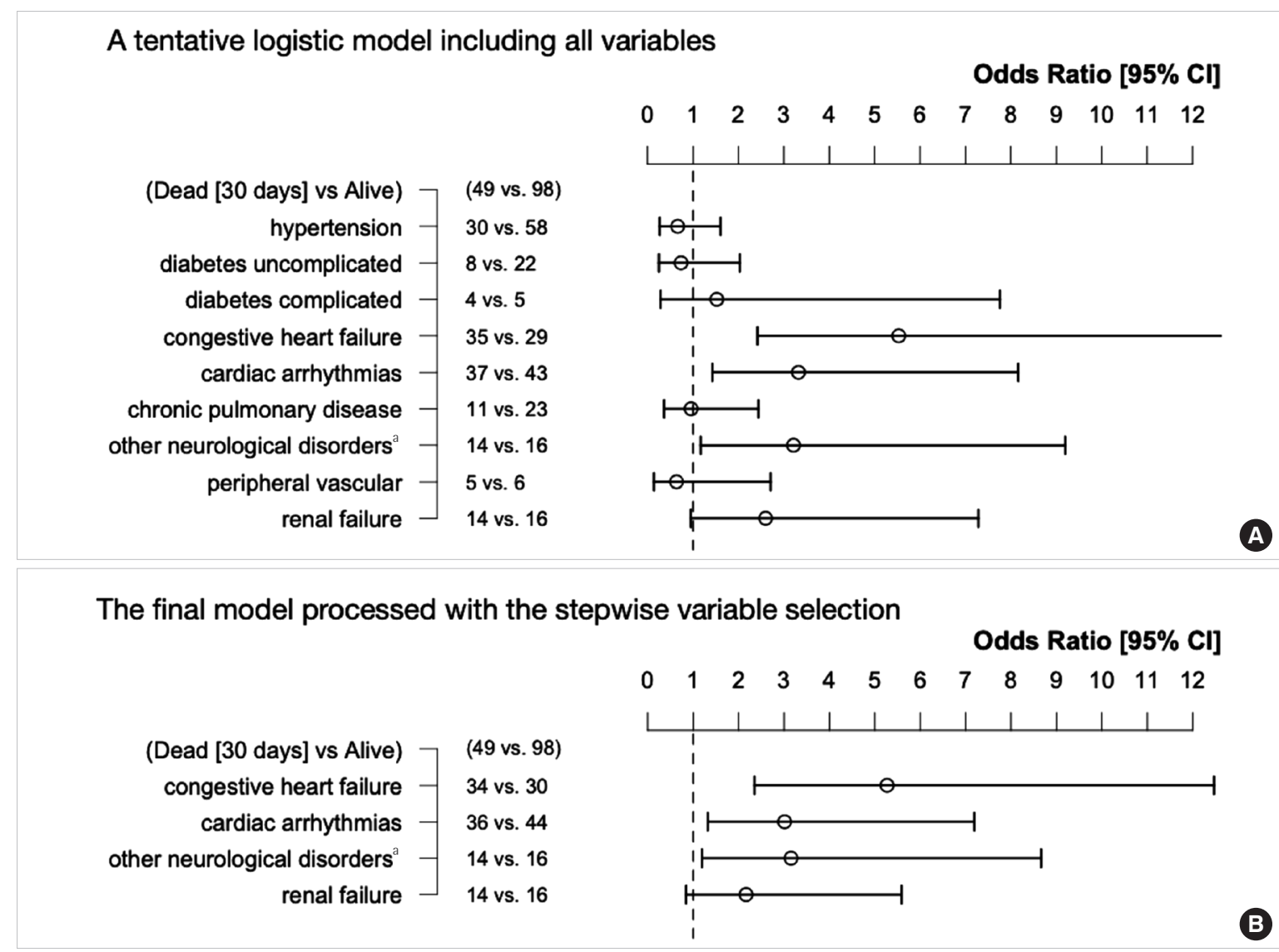

Figure 2. Forest plot of odds ratios for mortality, comparing case and control groups. (A) To explain 30-day mortality in patients after femur surgery, the nine candidate co-morbid factors are arbitrarily placed in the model. (B) Using the stepwise selection method, the factor list is shortened. The shortened model showed that congestive heart failure, cardiac arrhythmias, and other neurological disorders ${ }^{\mathrm{a}}$ are significant predictors of 30-day mortality. ${ }^{a}$ Other neurological disorders indicates neurological disorders other than paralysis, according to the Elixhauser Comorbidity measures [10]. Cl: confidence interval.

\section{DISCUSSION}

A femur fracture is a common trauma seen in older patients upon hospital admission and in surgical services. As society ages, the number of elderly patients undergoing femur fracture surgery is steadily increasing. Femur fractures are associated with a mortality rate of $6.2 \%$ to $8.3 \%$ within 30 days after surgery and $30 \%$ mortality within one year $[4,6,11]$. Even after discharge from the hospital, many patients are unable to return to their previous functional status and are taken into facilities such as nursing homes.

The reason for the high mortality rate among patients with this injury is that most patients are old and often have underlying medical diseases, such as cardiovascular disease and respiratory disease. The Charlson comorbidity index [12], geriatrics index of comorbidity [13], and the NHFS [9] are used to assess the risks of comorbidities. The well-known risk factors related to 30-day mortality indexed in the NHFS are anemia, older age, male sex, and the total number of comorbidities (cardiovascular, cerebrovascular, respiratory, renal diseases, diabetes, and systemic malignancy). We used anemia, age, and sex as variables for matching the control and case groups to evaluate the effect of individual comorbidities. Although the NHFS has been widely used and validated as a reliable tool in predicting 30-day mortality $[4,9,14]$, the influence of each disease is not taken into account by using a semi-quantitative value for the number of co-morbidities rather than individual values.

Our goal was to determine the effect of each individual disease on mortality. We selected nine conditions (from a set of 30 in the Elixhauser comorbidity measures) which were likely to be signifi-cant factors influencing mortality. Originally the Elixhauser comorbidity measures were developed to control preexisting disease or comorbidities when using an adminis- 
trative dataset for research [10]. Comorbidity was differentiated from complications by being defined as a clinical condition that existed before the hospitalization and was not related to the principal reason for the hospitali-zation. Therefore, only ICD-9-CM codes not related to the patient's diagnosis-related group (DRG) assignment were considered as comorbidities [10]. We chose the Elixhauser comorbidity measures for two reasons. First, the Elixhauser comorbidity measures provided a better prediction of in-hospital mortality after orthopedic surgery than the Charlson index, a well-known risk adjustment model [15]. Second, there are tables in the MIMIC-III database in which each patient's diagnosis is labeled with the ICD-9-CM code and DRG assignment.

The MIMIC-III is an open database created to provide free access to large amounts of digital health information. After completing the required training course, researchers are permitted to use the MIMIC-III in their clinical studies. Thus, researchers have the opportunity to use sophisti-cated electronic health records to predict clinical outcomes and validate published results in ICU settings.

Our study's patient population admitted to the ICU with multiple comorbidities was different from the general populations used in previous studies. Population characteristics explains the higher mortality (23.4\%) in our study, as compared to previous study results $(6.2 \%-8.3 \%)[4,6,11]$. Our study showed that congestive heart failure was the most important risk factor associated with patient mortality (OR, 4.99; 95\% CI, 2.18 to 12.06). Although less influential than congestive heart failure, cardiac arrhyth-mia and neurological disorders other than paralysis also showed significant results. These results can be used to predict the risk of a patient dying within 30 days of femur fracture surgery. Identify-ing these patient-specific mortality risk factors will allow caregivers to make informed decisions regarding aggressive treatment.

\section{CONFLICT OF INTEREST}

No potential conflict of interest relevant to this article was reported.

\section{ORCID}

Dohyung Kim

Hyunmin Jo

Younsuk Lee

Kyoung Ok Kim https://orcid.org/0000-0001-8245-8995 https://orcid.org/0000-0002-0957-8488 https://orcid.org/0000-0003-2488-5926 https://orcid.org/0000-0001-7509-3668

\section{AUTHOR CONTRIBUTIONS}

Conceptualization: KK. Data curation \& Formal analysis: YL, KK. Methodology: DK, KK. Visualization: DK, HJ. Writing - original draft: KK. Writing - review \& editing: all authors.

\section{REFERENCES}

1. Association of Anaesthetists of Great Britain and Ireland, Griffiths R, Alper J, Beckingsale A, Goldhill D, Heyburn G, et al. Management of proximal femoral fractures 2011: Association of Anaesthetists of Great Britain and Ireland. Anaesthesia 2012; 67:85-98.

2. Lee DK, Bang S, Lee S. Anesthetic considerations for surgical treatment of geriatric femur fracture. Anesth Pain Med 2019; 14:8-18.

3. Charlson M, Szatrowski TP, Peterson J, Gold J. Validation of a combined comorbidity index. J Clin Epidemiol 1994;47:124551.

4. Moppett IK, Parker M, Griffiths R, Bowers T, White SM, Moran CG. Nottingham Hip Fracture Score: longitudinal and multiassessment. Br J Anaesth 2012;109:546-50.

5. Nijmeijer WS, Folbert EC, Vermeer M, Slaets JP, Hegeman JH. Prediction of early mortality fol-lowing hip fracture surgery in frail elderly: the Almelo Hip Fracture Score (AHFS). Injury 2016;47:2138-43.

6. Dodd AC, Bulka C, Jahangir A, Mir HR, Obremskey WT, Sethi MK. Predictors of 30-day mortality following hip/pelvis fractures. Orthop Traumatol Surg Res 2016;102:707-10.

7. Kim BH, Lee S, Yoo B, Lee WY, Lim Y, Kim MC, et al. Risk factors associated with outcomes of hip fracture surgery in elderly patients. Korean J Anesthesiol 2015;68:561-7.

8. Johnson AE, Pollard TJ, Shen L, Lehman LW, Feng M, Ghassemi M, et al. MIMIC-III, a freely ac-cessible critical care database. Sci Data 2016;3:160035.

9. Maxwell MJ, Moran CG, Moppett IK. Development and validation of a preoperative scoring sys-tem to predict 30 day mortality in patients undergoing hip fracture surgery. $\mathrm{Br} \mathrm{J}$ Anaesth 2008;101:511-7.

10. Elixhauser A, Steiner C, Harris DR, Coffey RM. Comorbidity measures for use with administra-tive data. Med Care 1998; 36:8-27.

11. Wiles MD, Moran CG, Sahota O, Moppett IK. Nottingham Hip Fracture Score as a predictor of one year mortality in patients undergoing surgical repair of fractured neck of femur. Br J Anaesth 2011;106:501-4.

12. Charlson ME, Pompei P, Ales KL, MacKenzie CR. A new meth- 
od of classifying prognostic comor-bidity in longitudinal studies: development and validation. J Chronic Dis 1987;40:373-83.

13. Rozzini R, Frisoni GB, Ferrucci L, Barbisoni P, Sabatini T, Ranieri $\mathrm{P}$, et al. Geriatric Index of Comorbidity: validation and comparison with other measures of comorbidity. Age Ageing 2002;31:277-85.

14. Marufu TC, White SM, Griffiths R, Moonesinghe SR, Moppett
IK. Prediction of 30-day mortality after hip fracture surgery by the Nottingham Hip Fracture Score and the Surgical Outcome Risk Tool. Anaesthesia 2016;71:515-21.

15. Menendez ME, Neuhaus V, van Dijk CN, Ring D. The Elixhauser comorbidity method outper-forms the Charlson index in predicting inpatient death after orthopaedic surgery. Clin Orthop Relat Res 2014;472:2878-86. 